\title{
Efeito da suplementação do Momordica charantia $L$. em pacientes com Diabetes mellitus: Uma revisão sistemática
}

\author{
Effect of Momordica charantia L. supplementation on patients with Diabetes mellitus: A systematic \\ review
}

Efecto de la suplementación de Momordica charantia L. en pacientes con Diabetes mellitus: Una revisión sistemática

Edite Maria Morais do Nascimento ORCID: https://orcid.org/0000-0002-6519-688X Universidade Federal do Piauí, Brasil E-mail: editemaria214@gmail.com

Gabriela Lima de Araujo

ORCID: https://orcid.org/0000-0001-8366-7406 Universidade Federal do Piauí, Brasil E-mail: glima9700@gmail.com

Bruna Emanuele Pereira Cardoso ORCID: https://orcid.org/0000-0002-3863-1276 Universidade Federal do Piauí, Brasil E-mail: brunaemanuelec@hotmail.com

Stéfany Rodrigues de Sousa Melo ORCID: https://orcid.org/0000-0001-5308-3522 Universidade Federal do Piauí, Brasil E-mail: stefany.rsm@gmail.com

Maiara Jaianne Bezerra Leal Rios ORCID: https://orcid.org/0000-0003-2349-7235 Universidade Federal do Piauí, Brasil E-mail: maiararios2014@gmail.com Paulo Víctor de Lima Sousa ORCID: https://orcid.org/0000-0003-1486-0661 Universidade Federal do Piauí, Brasil E-mail: paulovictor.lima@hotmail.com

Guida Graziela Santos Cardoso ORCID: https://orcid.org/0000-0002-8639-7146 Universidade Federal do Piauí, Brasil E-mail: guida.cardoso18@gmail.com

Thaline Milany da Silva Dias ORCID: https://orcid.org/0000-0003-1734-4312 Universidade Federal do Piauí, Brasil E-mail: thatadias77@gmail.com Jennifer Beatriz Silva Morais ORCID: https://orcid.org/0000-0002-9055-7851 Universidade Federal do Piauí, Brasil

E-mail: jenniferbeatriz.morais@gmail.com

\begin{abstract}
Resumo
A diabetes mellitus (DM) é uma doença crônica, multifatorial que compreende um grupo heterogêneo de distúrbios metabólicos, classificando-se como um problema de saúde pública de dificil controle. O uso de plantas medicinais no controle de doenças vem ganhando bastante visibilidade devido a terapêtica, facilidade de acesso e casos clínicos bem sucedidos. Algumas espécies vegetais são empregadas para suprimir os sintomas causados pela diabetes mellitus em associação ou não com a medicina tradicional, dentre elas, o melão de São Caetano (Momordica charantia L.). A vigente revisão teve como intuito, analisar as principais pesquisas da utilização de Momordica charantia L. no tratamento de diabetes mellitus. Trata-se de uma revisão sistemática realizada com base em artigos originais publicados em inglês que foram selecionados nas bases de dados Pubmed, Cochrane Library, LILACS e ScienceDirect. Após a aplicação dos critérios de inclusão e exclusão, um total de 07 artigos foram finalmente selecionados para a pesquisa, onde observou-se que o $M$. charantia $L$. se mostrou uma alternativa com efeito antidiabético potencial no controle da diabetes mellitus.
\end{abstract}

Palavras-chave: Momordica charantia L; Resistencia à insulina; Diabetes; Medicina popular. 


\begin{abstract}
Diabetes mellitus (DM) is a chronic, multifactorial disease that comprises a heterogeneous group of metabolic disorders, being classified as a public health problem that is difficult to control. The use of medicinal plants in disease control has been gaining a lot of visibility due to therapy, easy access and successful clinical cases. Some plant species are used to suppress symptoms caused by diabetes mellitus in association or not with traditional medicine, among them, the São Caetano melón (Momordica charantia L.). The current review aimed to analyze the main researches on the use of Momordica charantia $L$. in the treatment of diabetes mellitus. This is a systematic review based on original articles published in English that were selected from the databases Pubmed, Cochrane Library, LILACS and ScienceDirect. After applying the inclusion and exclusion criteria, a total of 07 articles were finally selected for the research, where it was observed that $M$. charantia $L$. proved to be an alternative with a potential antidiabetic effect in the control of diabetes mellitus.
\end{abstract}

Keywords: Momordica charantia L; Insulin resistance; Diabetes; Popular medicine.

\title{
Resumen
}

La diabetes mellitus (DM) es una enfermedad crónica multifactorial que comprende un grupo heterogéneo de trastornos metabólicos, siendo catalogada como un problema de salud pública de difícil control. El uso de plantas medicinales en el control de enfermedades ha ido ganando mucha visibilidad debido a la terapia, la facilidad de acceso y los casos clínicos exitosos. Algunas especies vegetales se utilizan para suprimir los síntomas causados por la diabetes mellitus asociada o no a la medicina tradicional, entre ellas, el melón São Caetano (Momordica charantia L.). La presente revisión tuvo como objetivo analizar las principales investigaciones sobre el uso de Momordica charantia $L$. en el tratamiento de la diabetes mellitus. Se trata de una revisión sistemática basada en artículos originales publicados en inglés que fueron seleccionados de las bases de datos Pubmed, Cochrane Library, LILACS y ScienceDirect. Luego de aplicar los criterios de inclusión y exclusión, finalmente se seleccionaron un total de 07 artículos para la investigación, donde se observó que $M$. charantia $L$. resultó ser una alternativa con potencial efecto antidiabético en el control de la diabetes mellitus.

Palabras clave: Momordica charantia L; Resistencia a la insulina; Diabetes; Medicina popular.

\section{Introdução}

O diabetes mellitus (DM) compreende um grupo heterogêneo de distúrbios metabólicos que apresentam em comum a hiperglicemia, resultante da alteração, na ação ou na excreção da insulina pelas células beta pancreáticas, na qual essa alteração ocorre de forma parcial ou total, onde a partir disso irão classificar os tipos de diabetes, de acordo com a sua etiologia (Baade; Bueno, 2016). Trata-se de um problema de saúde de grande prevalência em nosso meio e considerado como uma das doenças de mais difícil controle, por ser crônica, sistêmica e multifatorial (Pereira; Abi, 2019).

O diabetes mellitus representa 5,2 milhões de mortes a nível global, com 252.806 mortes apenas nos Estados Unidos em 2015, segundo relatado pela Organização Mundial da Saúde (OMS), o diabetes mellitus de todos tipos cresceu excepcionalmente nas últimas décadas em todo o globo. A prevalência de DM aumentou de 108 milhões (4,7\%) em 1980 para 425 milhões (8,5\%) em 2017, e é previsto em 629 milhões em 2045 (Glovaci; Fan; Wong, 2019).

Cerca da metade dos adultos com diabetes mellitus viviam em países como a China, Índia, Estados Unidos, Indonésia e Brasil, em 2014. Em 2013, uma Pesquisa Nacional de Saúde de residentes brasileiros estimou a prevalência de DM em adultos de 6,2\% e em 2015, a Carga Global de Doenças (GBD) identificou o Brasil como tendo uma grande quantidade de doenças relacionadas ao diabetes (Guimarães et al., 2018).

A utilização popular de plantas medicinais é fundamentada em um conhecimento milenar, transmitido ao longo de gerações, e que muitas vezes constituía o único recurso em termos de cuidados médicos, curativos ou preventivos (Tresvenzol et al., 2006). De acordo com a Organização Mundial da Saúde (OMS), 85\% da população de países em desenvolvimento ainda recorrem a práticas antigas da medicina tradicional, devido principalmente ao difícil acesso ao sistema de saúde, com isso, sucedeu-se a necessidade da implementação dessa prática por profissionais de saúde afim de conservar essa cultura milenar e beneficiar a população com condutas já praticadas por tais (Brasil, 2006).

Segundo Alvarenga et al., (2017) há diversas espécies de plantas que são utilizadas na prática terapêutica de enfermidades humanas, sendo representativa a variedade empregada etnofarmacologicamente com fins antidiabéticos. Um 
exemplo disso, seria o Momodica charantia L. (M. charantia L.), pertencente à família Cucurbitaceae, também conhecido como melão de São Caetano, melão amargo, cabaço amargo, kugua, karela entre outras denominações. Sendo cultivadas em regiões tropicais e subtropicais como na China, Índia, Brasil, México, Tailândia, entre outros (Jia et al., 2017).

O $M$. charantia $L$. possui propriedades hipoglicêmicas, antioxidantes, antimicrobiano, anti-inflamatória, antiviral, anti-obesidade, imunomoduladoras, entre outros (Bakare et al., 2010). Além de possuir um rico valor nutricional em proteínas, carboidratos, vitaminas, fibras e minerais como o ferro, zinco, magnésio e sua polpa madura sendo fonte de licopeno caratenóide (Kwatra et al., 2016).Possui um efeito antidiabético, sendo muito utilizado como terapia alternativa para baixar os níveis de glicose no sangue em paciente com diabetes mellitus (Basch; Gabardi; Ulbricht, 2003). Estudos como o de Dans et al., (2007) apontam que seus frutos, extratos de folhas e sementes possuem um efeito hipoglicemiante.

Tendo em vista a relevância do diabetes mellitus como um problema de saúde pública, a importância e benefícios da medicina alternativa pela ingestão de fitoterápicos como o $M$. charantia $L$. no controle glicêmico nesses indivíduos, assim como a inconsistência dos dados sobre esses aspectos, torna-se relevante a realização de estudos de revisão que possam reunir maiores informações, para melhores esclarecimentos e fomentar esses dados.

Desse modo, o objetivo deste trabalho foi descrever, em uma revisão sistemática, os desfechos de ensaios clínicos relativos à suplementação com $M$. charantia $L$. e resistência à insulina em pacientes com diabetes mellitus.

\section{Metodologia}

\section{Estratégia de busca e critérios de elegibilidade}

A pesquisa dos artigos nas bases de dados foi realizada por duas autoras de forma independente (EMMN e GLA). As pesquisas realizadas pelas referidas autoras foram comparadas, sendo verificada equivalência na busca e seleção dos artigos. A pesquisa dos artigos foi realizada nas bases de dados Pubmed, Cochrane Library, LILACS e Science Direct. As palavras chave (Momordica charantias OR Bitter Melon OR Bitter Gourd) AND (diabetes mellitus OR diabetic OR Resistance, Insulin OR Insulin Sensitivity OR Sensitivity, Insulin) foram utilizadas para o levantamento bibliográfico.

Primeiramente os artigos foram filtrados através da leitura dos títulos, em seguida dos resumos, nos quais foram incluídos ensaios clínicos randomizados, duplo cego, placebo controlados, caso-controle publicados em inglês, sem limite de ano de publicação, que avaliaram o efeito da suplementação com Momordica charantia sobre a resistência à insulina em humanos e animais com diabetes, sem restrição de gênero, idade e etnia. Não foram incluídos nessa revisão relatos de caso, estudos transversais e artigos de revisão.

Informações relevantes foram coletadas dos artigos dessa revisão, incluindo autores, ano de publicação, local de realização da pesquisa, tamanho da amostra, sexo e idade da população estudada, dose e duração da suplementação, grupo controle ou placebo, métodos de avaliação dos parâmetros de resistência à insulina.

As recomendações do Preferred Reporting Items for Systematic Reviews and Meta-Analyses (PRISMA) foram utilizadas como guia para a escrita cientifica do artigo.

Os critérios PICOS (Participantes, Intervenções, Comparações, Resultados e Desenho do estudo) foram utilizados para definir a questão de pesquisa (Tabela 1). Com a ajuda da ferramenta MESH (Medical Subject Headings), os seguintes descritores foram usados: "Momordica charantia", "suplementação", "diabetes". 
Tabela 1. PICOS criteria for inclusion of studies.

\begin{tabular}{|c|c|}
\hline Parâmetros & Crohn \\
\hline Participantes/população & $\begin{array}{l}\text { Humanos com diabetes } \\
\text { mellitus e ratos }\end{array}$ \\
\hline Intervenção & $\begin{array}{l}\text { Suplementação } \quad \text { com } \\
\text { Momordica charantia } L\end{array}$ \\
\hline Comparação & $\begin{array}{l}\text { Com placebo ou controle } \\
\text { ou suplementação }\end{array}$ \\
\hline Resultados & $\begin{array}{lcc}\text { Mudanças no curso da } \\
\text { doença; } & \text { atividade da } \\
\text { doença; } & \text { parâmetros } \\
\text { bioquímicos; marcadores } \\
\text { de infamação }\end{array}$ \\
\hline Design de estudos & Ensaios clínicos \\
\hline
\end{tabular}

Fonte: Autores.

\section{Avaliação do Risco de Vieses}

A Cochrane Risk of Bias Tool foi usada para avaliar o risco de viés dos ensaios clínicos incluídos nesse estudo.

A (Figura 1) identifica o quantitativo final obtido com base nos requisitos pré-definidos na metodologia. No final, 7 artigos foram usados na pesquisa.

Figura 1. Fluxograma do processo de seleção dos artigos.

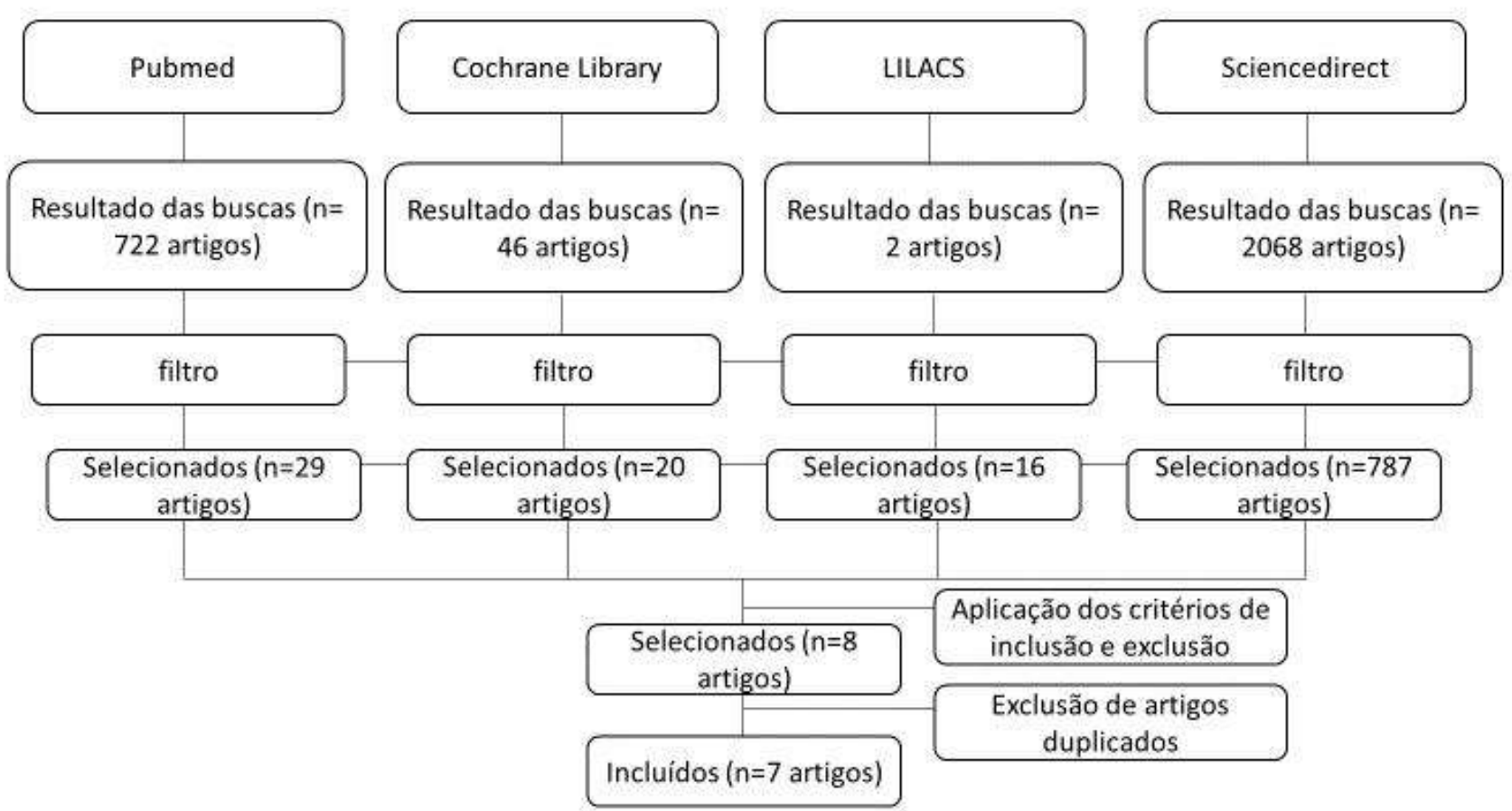

Fonte: Autores. 


\section{Resultados e Discussão}

\section{Artigos Selecionados}

Um total de 07 artigos foram identificados por meio de pesquisa nas bases de dados PubMed ( $\mathrm{n}=722$ ), Cochrane Library ( $\mathrm{n}=46)$, Lilacs $(\mathrm{n}=2)$ e Science Direct $(\mathrm{n}=2068)$. Após o processo de triagem, remoção de artigos duplicados e análises dos critérios de inclusão, 07 artigos foram identificados como elegíveis para esta revisão sistemática. As (Tabelas 2 e 3 ) mostram as características dos artigos incluídos, realizados em humanos e ratos, respectivamente.

Tabela 2. Características dos estudos em humanos incluídos nessa revisão.

\begin{tabular}{|c|c|c|c|c|c|c|c|c|}
\hline \multirow{2}{*}{$\begin{array}{l}\text { Autores/ } \\
\text { ano } \\
\text { de } \\
\text { publicação }\end{array}$} & \multicolumn{4}{|c|}{ Humanos } & \multicolumn{3}{|c|}{ Suplementação } & \multirow{2}{*}{$\begin{array}{l}\text { Principais } \\
\text { Desfechos }\end{array}$} \\
\hline & País & $\begin{array}{c}\text { (n) } \\
\text { participantes }\end{array}$ & Gênero & $\begin{array}{l}\text { Idade } \\
\text { (anos) }\end{array}$ & Dose & Medicamentos & Duração & \\
\hline $\begin{array}{l}\text { Leatherdale } \\
\text { et al. (1981) }\end{array}$ & Ásia & $\begin{array}{c}08- \\
\text { Suplemento } \\
01 \text { - Controle }\end{array}$ & $\mathrm{M} / \mathrm{F}$ & $*$ & $\begin{array}{c}50 \mathrm{ml}-\text { Suco } \\
\text { de Karela } \\
0,23 \mathrm{~kg}- \\
\text { Karela Frita }\end{array}$ & $\begin{array}{l}\text { Clorpropamida, } \\
\text { tolbutamida, } \\
\text { glienclanida, } \\
\text { glicedina. }\end{array}$ & 87 dias & $\begin{array}{c}\text { Melhora da } \\
\text { tolerância à } \\
\text { glicose; } \\
\text { [ ] de insulina } \\
\text { não } \\
\text { aumentada; } \\
\downarrow \text { HbA1-c; } \\
\text { Propriedade } \\
\text { hipoglicêmica. }\end{array}$ \\
\hline $\begin{array}{l}\text { Ranhman } \\
\text { et } \\
\text { al. }(\mathbf{2 0 1 5})\end{array}$ & Paquistão & $\begin{array}{c}95- \\
\text { Suplemento } \\
112 \text { - Placebo }\end{array}$ & $\mathrm{M} / \mathrm{F}$ & $30-70$ & $\begin{array}{l}32 \text { pessoas - } \\
2 \mathrm{~g} / \mathrm{dia} \\
33 \mathrm{pessoas} \mathrm{-} \\
4 \mathrm{~g} / \mathrm{dia}\end{array}$ & $\begin{array}{c}30 \text { pessoas - } \\
2,5 \mathrm{~g} / \mathrm{dia} \\
\text { (glibenclamida) }\end{array}$ & $\begin{array}{c}10 \\
\text { semanas }\end{array}$ & $\begin{array}{c}\downarrow \text { HbA1-c; } \\
\downarrow \text { FPG (> no } \\
\text { grupo 3); } \\
\downarrow \text { média no } \\
\text { PAS; } \\
\text { Hipoglicemia. }\end{array}$ \\
\hline $\begin{array}{l}\text { Kumari; } \\
\text { Dash; } \\
\text { Behera, } \\
\text { (2018) }\end{array}$ & Índia & $\begin{array}{c}50- \\
\text { Suplemento } \\
25-\text { Controle }\end{array}$ & $\mathrm{M} / \mathrm{F}$ & $40-60$ & $\begin{array}{c}\text { Grupo A - 1g, } \\
\text { Grupo B - } \\
\text { 1,5g ambos } \\
\text { adicionados } \\
\text { de } \\
\text { antidiabéticos } \\
\text { orais } \\
\text { (metformina e } \\
\text { glibendamida) }\end{array}$ & $\begin{array}{l}\text { Metformina e } \\
\text { glibendamida }\end{array}$ & $\begin{array}{c}8 \\
\text { semanas }\end{array}$ & $\begin{array}{c}\downarrow \text { níveis de } \\
\text { açúcar no } \\
\text { sangue; } \\
\downarrow \text { HbA1-c; } \\
\text { Melhora a } \\
\text { sensibilidade à } \\
\text { insulina; } \\
\text { Melhora a RI. }\end{array}$ \\
\hline
\end{tabular}

HbA1-c: hemoglobina glicada; FPG: glicemia de jejum; PAS: pressão arterial sistólica; RI: resistência à insulina. Fonte: Autores. 
Tabela 3. Características dos estudos em animais incluídos nessa revisão.

\begin{tabular}{|c|c|c|c|c|c|c|c|c|}
\hline \multirow{2}{*}{$\begin{array}{c}\text { Autores/ } \\
\text { ano de } \\
\text { publicação }\end{array}$} & \multicolumn{4}{|c|}{ Animais } & \multicolumn{3}{|c|}{ Suplementação } & \multirow{2}{*}{$\begin{array}{l}\text { Principais } \\
\text { Desfechos }\end{array}$} \\
\hline & País & $\begin{array}{c}\text { (n) } \\
\text { participantes }\end{array}$ & Gênero & Espécie & Dose & Medicamentos & Duração & \\
\hline $\begin{array}{l}\text { Jing et al. } \\
(2020)\end{array}$ & China & $\begin{array}{c}30- \\
\text { Suplemento } \\
10 \text { - Controle }\end{array}$ & $\mathrm{M}$ & Wistar & $\begin{array}{l}\text { Alta dose - } \\
\text { 400mg, } \\
\text { Média - } \\
\text { 200mg, } \\
\text { Baixa - } \\
100 \mathrm{mg}\end{array}$ & $*$ & $\begin{array}{c}4 \\
\text { semanas }\end{array}$ & $\begin{array}{c}\downarrow \text { \FBG; } \\
\uparrow F I N S ; \\
\uparrow I S I ; \\
\downarrow \text { RI; } \\
\downarrow \text { açúcar no } \\
\text { sangue em } \\
\text { jejum, } \\
\text { Efeito anti- } \\
\text { diabético. }\end{array}$ \\
\hline $\begin{array}{c}\text { Yang et al. } \\
(2014)\end{array}$ & $\begin{array}{c}\text { Correia } \\
\text { do Sul }\end{array}$ & $\begin{array}{c}20- \\
\text { Suplemento } \\
07 \text { - Placebo }\end{array}$ & $\mathrm{M}$ & OLEFT & $\begin{array}{c}\text { Pó liofilizado } \\
\text { do } \\
\text { BM } \\
(\mathrm{HF}+1 \% \\
\text { BM }) ; \\
(\mathrm{HF}+3 \% \\
\text { BM })\end{array}$ & $*$ & $\begin{array}{c}6 \\
\text { semanas }\end{array}$ & $\begin{array}{c}\downarrow \text { Insulina de } \\
\text { jejum } \\
\downarrow \text { Glicose de } \\
\text { jejum } \\
\downarrow \text { HOMA-IR } \\
\text { (mais baixos } \\
\text { em 3\%) }\end{array}$ \\
\hline $\begin{array}{l}\text { Xu et al. } \\
\text { (2015) }\end{array}$ & China & $\begin{array}{c}40 \text { - } \\
\text { Suplemento } \\
50 \text { - Placebo }\end{array}$ & $\mathrm{M} / \mathrm{F}$ & Kunming & $\begin{array}{c}\text { MCP na forma } \\
\text { de mucilagem } \\
\text { Ratos normais: } \\
\text { Controle } \\
\text { normal * } \\
\text { Controle } \\
\text { positivo - } \\
200 \mathrm{mg} / \mathrm{kg} \\
\mathrm{MCP}- \\
200 \mathrm{mg} / \mathrm{kg} \\
\text { ratos } \\
\text { diabéticos } \\
\text { induzidos por } \\
\text { aloxana: } \\
\text { Controle } \\
\text { normal } * \\
\text { Controle } \\
\text { diabético } * \\
\text { Controle } \\
\text { positivo - } \\
\text { pílula } 200 \\
\text { mg/kg } \\
\text { MCP-L- } \\
100 \mathrm{mg} / \mathrm{kg} \\
\mathrm{MCP}-\mathrm{M}- \\
200 \mathrm{mg} / \mathrm{kg} \\
\mathrm{MCP}-\mathrm{H}- \\
300 \mathrm{mg} / \mathrm{kg} \\
\end{array}$ & Xiaoke & 28 dias & $\begin{array}{c}\downarrow \text { Glicose de } \\
\text { jejum }\end{array}$ \\
\hline $\begin{array}{c}\text { Wang et } \\
\text { al. } \\
(2014)\end{array}$ & China & $\begin{array}{c}08 \mathrm{KK} / \mathrm{HIJ} \\
08 \mathrm{ICR}- \\
\text { Suplemento } \\
08 \mathrm{KK} / \mathrm{HIJ} \\
08 \mathrm{ICR} \text { - } \\
\text { Placebo }\end{array}$ & M & $\begin{array}{c}\mathrm{KK} / \mathrm{HIJ} \\
\mathrm{ICR}\end{array}$ & $\begin{array}{l}\text { Pó de MC } \\
200 \mathrm{mg} / \mathrm{kg}\end{array}$ & Tolbutamida & $\begin{array}{c}8 \\
\text { semanas }\end{array}$ & $\begin{array}{c}\text { KK/HIJ } \\
\downarrow \text { níveis } \\
\text { plasmáticos } \\
\text { de insulina } \\
\uparrow \text { GLUT4 } \\
\uparrow I R S-1 \\
\uparrow \text { ISI } \\
\text { ICR } \\
\downarrow \text { Glicose de } \\
\text { jejum }\end{array}$ \\
\hline
\end{tabular}


FBG: glicemia de jejum; FINS: insulina de jejum; ISI: sensibilidade à insulina; RI: resistência à insulina; HOMA-IR: Homeostasis Model Assessment for Insulin Resistance; MCP: polissacarídeo dos frutos de Momordica charantia; MC: Momordica charantia; BM: melão amargo. HF: dieta rica em gordura. Fonte: Autores.

Com base na avaliação dos 07 estudos incluídos nesta revisão, observou-se que 3 dos ensaios clínicos foram realizados com pessoas adultas, de ambos os sexos, em três países diferentes, nas quais as doses de suplementação do $M$. charantia $L$. variaram de 1,5 a 23,0 g/dia. A duração dos testes foi entre 8 a 12 semanas, onde todos os estudos avaliaram a hemoglobina glicada $(\mathrm{HbA1}-\mathrm{c})$ dentre outros parâmetros como a tolerância à glicose, glicemia de jejum, resistência à insulina $\mathrm{e}$ concentrações de insulina.

Os outros quatro estudos foram realizados em ratos de ambos os sexos e de diferentes espécies sendo a maioria na China e um na Coréia do Sul, na qual o M. charantia L. foi administrado entre 100 a $400 \mathrm{mg} / \mathrm{dia}$, nas formas de mucilagem e pílula com uma duração entre 4 a 8 semanas. Sendo avaliados insulina de jejum, sensibilidade à insulina e o Homeostasis Model Assessment For Insulin Resistance (HOMA-IR).

Qualidade metodológica dos estudos incluídos

A avaliação da qualidade metodológica dos estudos incluídos nesta revisão está apresentada na (Tabela 4). Os estudos satisfazem a maioria dos critérios estabelecidos para avaliar a qualidade metodológica, segundo a Cochrane Risk of Bias Tool.

Tabela 4. Qualidade metodológica dos estudos incluídos;

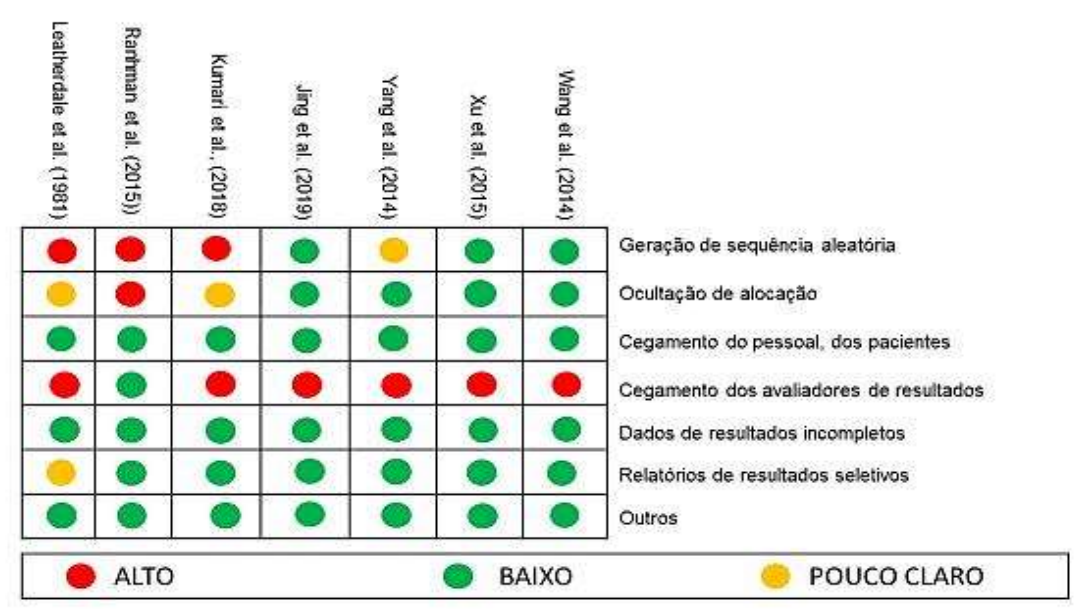

Efeito da suplementação com M. charantia L. sobre a RI em diabéticos. Fonte: Autores.

Acerca da insulina, suas concentrações não foram aumentadas e houve uma melhora na sensibilidade e resistência à insulina. Estudos como o de Kumari et al., (2018), mostraram que os níveis de resistência à insulina não foram consideravelmente alterados, no entanto com a administração de 1,5g do Momordica charantia L., observou-se a melhora na RI, sugerindo o efeito positivo do Momordica charantia $L$. sob as funções das células beta pancreáticas.

Nos demais estudos realizados em humanos, não foram apresentados parâmetros sobre a resistência à insulina, porém Leatherdale et al., (1981), apresentou a concentração de insulina não aumentada após suplementação com o suco e a fruta frita além da melhora na tolerância à glicose e redução da hemoglobina glicada (HbA1-c), que foi o mesmo apresentado por Ranhman et al., (2015), que usou diferentes doses de suplementação e ainda o medicamento, glibenclamida, que foi o que relatou uma maior redução na glicemia de jejum.

Quanto aos efeitos da suplementação em ratos, foram aplicadas a cinco espécies diferentes onde houve uma redução na resistência à insulina, nos seus níveis plasmáticos e na insulina em jejum. Jing et al (2020), distribuíram baixas, médias e altas doses de 100, 200 e $400 \mathrm{mg}$ respectivamente, nos quais os grupos que receberam alta e média dose apresentaram aumento 
na sensibilidade à insulina e na glicemia de jejum, e este estudo apresentou também a redução na resistência à insulina e no açúcar no sangue em jejum.

No estudo de Yang et al., (2014) foram utilizadas concentrações de 1\% e 3\% de pó liofilizado de M. charantia L. em ratos diabéticos, onde foi constatado a redução de insulina de jejum, glicose de jejum e HOMA-IR menores na concentração de $3 \%$, indicando que o $M$. charantia $L$. tem a capacidade de aumentar a sensibilidade à insulina.

Já no estudo de Wang et al., (2014), onde foram utilizadas duas espécies de camundongos, ICR para avaliar o extrato rico em charantina de $M$. charantia $L$. na diabetes tipo 1 e KK/HIJ para avaliar o extrato na diabetes tipo 2, observou-se melhora no quadro de resistência à insulina em ratos $\mathrm{KK} / \mathrm{HIJ}$, indicando que o extrato de $M$. charantia $L$. tem potencial para aumentar a sensibilidade à insulina em pacientes com diabetes tipo $2 \mathrm{em}$ vez de proteger os pacientes com diabetes tipo 1 contra a disfunção das células $\beta$. O estudo de Xu et al., (1015), não apresentou parâmetros sobre a resistência à insulina, somente sobre redução da glicose em jejum. Um polissacarídeo solúvel em água extraído dos frutos de $M$. charantia L. nas concentrações de $100 \mathrm{mg} / \mathrm{kg}, 200 \mathrm{mg} / \mathrm{kg}$ e $300 \mathrm{mg} / \mathrm{kg}$ foram fornecidas a ratos diabéticos induzidos por aloxano. A concentração de $300 \mathrm{mg} / \mathrm{kg}$ reduziu os níveis de açúcar no sangue.

Quanto ao efeito apresentado na compilação de dados, observou-se que a maioria dos estudos apresentou redução da resistência à insulina. Um ponto importante a ser salientado nesta discussão, diz respeito às discordâncias acerca das doses, bem como a forma de suplementação do $M$. charantia $L$. e o tempo de suplementação, que podem ter influenciado alguns parâmetros utilizados para avaliar o metabolismo da glicose.

O estudo de Dans et al. (2007) mostrou a eficácia no suco do M. charantia L. e na fruta na forma frita, no que diz respeito a melhora da tolerância à glicose e em termos de propriedades hipoglicêmicas. Como em um estudo envolvendo 9 pacientes diabéticos que apresentou melhora significativa na tolerância à glicose, após o consumo diário da fruta frita e o consumo do suco da polpa.

De acordo com o mesmo estudo, constatou-se a redução da glicemia em jejum, porém no grupo na qual houve a administração de glibenclamida, esse medicamento tem a ação principal de aumentar a secreção da insulina, diminuindo a produção hepática de glicose, aumentando a sensibilidade dos tecidos à insulina (Silva et al., 2007). Houve também a redução da Pressão Arterial Sistólica (PAS) significativa no grupo no qual foi administrado o extrato aquoso do melão amargo.

O M. charantia $L$. tem seu efeito hipoglicemiante devido ao seu papel na resistência e secreção da insulina, portanto no estudo de Kumari et al., (2018), onde existiram 3 grupos, 2 recebendo 1,0 e 1,5g do M. charantia L. respectivamente, constatou-se que o grupo que recebeu uma dose maior é mais eficaz no controle do perfil glicêmico. Com isso o efeito benéfico nos níveis de açúcar no sangue é atribuído a capacidade do M. charantia L.de preservar a integridade estrutural das ilhotas pancreáticas e liberação de hormônios (Hafizur; Kabir; Chishti, 2011).

Outro ponto importante a se avaliar, diz respeito aos locais em que os estudos foram realizados, no continente asiático. Segundo Walters e Decker-Walters (1988), a planta é cultivada principalmente na Índia, China, África Oriental e Central e da América do Sul, que por ser o centro de origem, colaborou com os pontos de pesquisa, pois há uma maior disponibilidade de material vegetal para estudo afim de avaliar as suas propriedades medicinais.

Ao analisar os estudos feitos em ratos na presente revisão, constatou-se que em todos os níveis de glicose em jejum foram diminuídos. Segundo Bhutkar; Bhise (2012) e Percin et al., (2018), os principais compostos responsáveis pela ação antihiperglicêmica do $M$. charantia $L$. são triterpenos proteicos, esteroides, alcalóides e fenólicos que inibem a $\alpha$-amilase e aglucosidade, responsáveis pela quebra do amido em açúcares. Portanto, os inibidores dessas enzimas podem diminuir a digestão dos carboidratos e consequentemente os valores glicêmicos (Fernandes et al., 2007).

É de essencial importância que nos atentemos para as diferentes formas de preparo as quais o M. charantia $L$. foi inserido nas referentes pesquisas. Segundo Fernandes et al., (2007) e Fuangchan et al., (2011), todas as partes da planta são 
utilizadas para diminuição dos níveis de glicose sanguínea e existem diversas variedades de métodos de preparo destinados ao tratamento de diabetes mellitus, pois os compostos fenólicos do M. charantia L. possuem propriedades antidiabéticas (Vinayagam; Jayachandran; Xu, 2016) e antioxidantes (Wu; Ng, 2008).

A maioria das pesquisas inclusas nesta revisão foram realizadas em animais. De acordo com Malinoski et al., (2010), a indução farmacológica através da administração de agentes citotóxicos que induzem a diabetes mellitus, possui baixo custo e permite alterações diretas no metabolismo basal dos animais, o que os torna um grupo com potencial mais econômico.

A presente revisão veio nos apontar que todos os desfechos dos estudos selecionados foram similares, indicando que o M. charantia $L$. e seus compostos bioativos apresentam efeito potencial no controle da diabetes mellitus.

Uma limitação considerável corresponde a artigos não terem sido incluídos nesta revisão, devido não apresentarem parâmetros como avaliação da resistência à insulina, como o HOMA-IR, e também por tratarem da relação do Momordica charantia L. com diabetes, porém não terem sido realizados com pacientes com diabetes mellitus tipo 2.

\section{Conclusão}

De acordo com os resultados evidenciados na presente revisão sistemática, pôde-se observar que a Momordica charantia $L$. possui efeitos antidiabéticos benéficos atuantes na resistência à insulina, glicemia de jejum, bem como em outros parâmetros importantes como hemoglobina glicada e HOMA-IR em indivíduos com diabetes mellitus. No entanto, torna-se necessário que mais estudos sejam realizados afim de padronizar as doses e tempo de suplementação.

\section{Referências}

Alvarenga, C. F., de Lima, K. M. N., Mollica, L. R., Azeredo, L. O., \& de Carvalho, C. (2017). Uso de plantas medicinais para o tratamento do diabetes mellitus no Vale do Paraíba-SP. Revista Ciência e Saúde On-line, 2(2).

Baade, R. T. W., \& Bueno, E. (2016). Coconstrução da autonomia do cuidado da pessoa com diabetes. Interface-Comunicação, Saúde, Educação, 20, 941951.

Bakare, R. I., Magbagbeola, O. A., \& Okunowo, O. W. (2010). Nutritional and chemical evaluation of Momordica charantia. Journal of Medicinal Plants Research, 4(21), 2189-2193.

Basch, E., Gabardi, S., \& Ulbricht, C. (2003). Bitter melon (Momordica charantia): a review of efficacy and safety. American Journal of Health-System Pharmacy, 60(4), 356-359.

Bhutkar, M. A., \& Bhise, S. B. (2012). In vitro assay of alpha amylase inhibitory activity of some indigenous plants. Int. J. Chem. Sci, 10(1), 457-462.

Brasil (2006). Política nacional de plantas medicinais e fitoterápicos. Ministério da Saúde, Secretaria de Ciência, Tecnologia e Insumos Estratégicos, Departamento de Assistência Farmacêutica. Brasília.

Dans, A. M. L., Villarruz, M. V. C., Jimeno, C. A., Javelosa, M. A. U., Chua, J., Bautista, R., \& Velez, G. G. B. (2007). The effect of Momordica charantia capsule preparation on glycemic control in type 2 diabetes mellitus needs further studies. Journal of clinical epidemiology, 60(6), 554-559.

Fernandes, N. P., Lagishetty, C. V., Panda, V. S., \& Naik, S. R. (2007). An experimental evaluation of the antidiabetic and antilipidemic properties of a standardized Momordica charantia fruit extract. BMC complementary and alternative medicine, 7(1), 1-8.

Fuangchan, A., Sonthisombat, P., Seubnukarn, T., Chanouan, R., Chotchaisuwat, P., Sirigulsatien, V., \& Haines, S. T. (2011). Hypoglycemic effect of bitter melon compared with metformin in newly diagnosed type 2 diabetes patients. Journal of ethnopharmacology, 134(2), 422-428.

Glovaci, D., Fan, W., \& Wong, N. D. (2019). Epidemiology of diabetes mellitus and cardiovascular disease. Current cardiology reports, 21(4), 1-8.

Guimarães, R. A., Morais Neto, O. L. D., Souza, M. R. D., Cortez-Escalante, J. J., Santos, T. A. D. P., Rosso, C. F. W., \& Maia, L. G. (2019). Epidemiology of Self-Reported Diabetes Mellitus in the State of Maranhão, Northeastern Brazil: Results of the National Health Survey, 2013. International journal of environmental research and public health, 16(1), 47.

Hafizur, R. M., Kabir, N., \& Chishti, S. (2011). Modulation of pancreatic $\beta$-cells in neonatally streptozotocin-induced type 2 diabetic rats by the ethanolic extract of Momordica charantia fruit pulp. Natural Product Research, 25(4), 353-367.

Jia, S., Shen, M., Zhang, F., \& Xie, J. (2017). Recent advances in Momordica charantia: functional components and biological activities. International journal of molecular sciences, 18(12), 2555.

Jiang, S., Xu, L., Xu, Y., Guo, Y., Wei, L., Li, X., \& Song, W. (2020). Antidiabetic effect of Momordica charantia saponins in rats induced by high-fat diet combined with STZ. Electronic Journal of Biotechnology, 43, 41-47. 
Kumari, S., Dash, I., \& Behera, K. K. (2018). Therapeutic Effect of Momordica charantia on Blood Glucose, Lipid Profile and Oxidative Stress in Type 2 Diabetes Mellitus Patients: A Randomised Controlled Trial. Journal of Clinical \& Diagnostic Research, 12(9).

Kwatra, D., Dandawate, P., Padhye, S., \& Anant, S. (2016). Bitter melon as a therapy for diabetes, inflammation, and cancer: a panacea? Current Pharmacology Reports, 2(1), 34-44.

Leatherdale, B. A., Panesar, R. K., Singh, G., Atkins, T. W., Bailey, C. J., \& Bignell, A. H. (1981). Improvement in glucose tolerance due to Momordica charantia (karela). British medical jornal. 282(6279), 1823-1824.

Malinoski, B., Cain, J. P., Amaral, L. A., Antonovicz, S., Bernardi, L. (2020). Modelos experimentais para estudos em nutrição: hipertensão arterial e diabetes mellitus. Visão Acadêmica, Curitiba. 17(4).

Percin, P. S., Inanli, O., Karakaya, S. (2018). In vitro $\alpha$-amylase and $\alpha$-glucosidase inhibitory activities of bitter melon (Momordica charantia) with different stage of maturity. International Journal of Nutrition and Food Engineering. 12(1).

Pereira, F. K., \& Abi, R. C. D. (2019). Complicações do Diabetes Mellitus. International Journal of Health Management, 5(1).

Rahman, I. U., Khan, R. U., Rahman, K. U., \& Bashir, M. (2015). Lower hypoglycemic but higher antiatherogenic effects of bitter melon than glibenclamide in type 2 diabetic patients. Nutrition journal, 14(1), 1-7.

Silva, J. C., Heinen, A., Scheidt, M. B., Marcondes, M. A. D. O., \& Bertini, A. M. (2007). Tratamento do diabetes mellitus gestacional com glibenclamida: fatores de sucesso e resultados perinatais. Revista Brasileira de Ginecologia e Obstetrícia, 29(11), 555-560.

Tresvenzol, L. M. F., Paula, J. R. D., Ricardo, A. F., Ferreira, H. D., \& Zatta, D. T. (2006). Estudo sobre o comércio informal de plantas medicinais em Goiânia e cidades vizinhas. Revista Eletrônica de Farmácia. 3(1), 23-8.

Vinayagam, R., Jayachandran, M., \& Xu, B. (2016). Antidiabetic effects of simple phenolic acids: a comprehensive review. Phytotherapy research, 30(2), 184-199.

Walters, W., Decker-Walters, D. S. (1988). Notes on Economic plants. Econ. Botan. 42(2), 286-292.

Wang, H. Y., Kan, W. C., Cheng, T. J., Yu, S. H., Chang, L. H., \& Chuu, J. J. (2014). Differential anti-diabetic effects and mechanism of action of charantinrich extract of Taiwanese Momordica charantia between type 1 and type 2 diabetic mice. Food and Chemical Toxicology, 69, 347-356.

Wu, S. J., \& Ng, L. T. (2008). Antioxidant and free radical scavenging activities of wild bitter melon (Momordica charantia Linn. var. abbreviata Ser.) in Taiwan. LWT-Food Science and Technology, 41(2), 323-330.

Xu, X., Shan, B., Liao, C. H., Xie, J. H., Wen, P. W., \& Shi, J. Y. (2015). Anti-diabetic properties of Momordica charantia L. polysaccharide in alloxaninduced diabetic mice. International journal of biological macromolecules, 81, 538-543.

Yang, S. J., Choi, J. M., Park, S. E., Rhee, E. J., Lee, W. Y., Oh, K. W., \& Park, C. Y. (2015). Preventive effects of bitter melon (Momordica charantia) against insulin resistance and diabetes are associated with the inhibition of NF- $\mathrm{BB}$ and JNK pathways in high-fat-fed OLETF rats. The Journal of Nutritional Biochemistry, 26(3), 234-240. 